\title{
ATLAS and CMS Upgrade Plans
}

\author{
Ulrich Parzefall on behalf of the ATLAS and CMS Collaborations. ${ }^{* \dagger}$ \\ Physikalisches Institut, \\ Albert-Ludwigs Universität Freiburg, \\ Hermann-Herder-Strasse 3, \\ D-79104 Freiburg \\ E-mail: Ulrich.Parzefallecern.ch
}

It is foreseen to significantly increase the luminosity of the LHC through upgrades leading towards HL-LHC (High Luminosity LHC) operation in order to harvest the maximum physics potential of this facility. Especially for the Phase-II Upgrade foreseen for 2022, this will mean unprecedented track densities from the high instantaneous luminosity, and extremely high radiation levels proportional to the total integrated luminosity over the lifetime of the detectors. In addition, the existing detectors will have accumulated a significant radiation dose already by the time of the long shutdown, before the HL-LHC starts. In order to keep the performance roughly at present levels in the challenging HL-LHC conditions, major upgrades of the detectors are planned. In particular the inner detector systems of both experiments will need to be replaced to maintain the tracking, vertexing and b-tagging capabilities. For this replacement, all-silicon central trackers are being studied in the two general purpose LHC experiments ATLAS and CMS. This paper describes the layout and technical details of the planned silicon detector systems for both experiments. Due to space constraints, only a limited selection of individual upgrade details can be presented here. Given the B-Physics centered focus of the Beauty conference series, this paper largely concentrates on upgrades to the silicon-based inner detector components that are vital to e.g. the identification of secondary vertices.

14th International Conference on B-Physics at Hadron Machines April 8-12, 2013

Bologna, Italy

\footnotetext{
* Speaker.

${ }^{\dagger}$ The author is member of the ATLAS Collaboration.
} 


\section{Introduction}

The two general-purpose LHC[1] experiments ATLAS[2] and CMS[3] will undergo a series of detector upgrades aligned to the timeline of the LHC accelerator chain consolidation and upgrade. The LHC schedule foresees three long shutdowns on the way to the goal of ultimately integrating $3000 \mathrm{fb}^{-1}$. In each of these shutdowns, a large number of different detector upgrades will be performed, with the major upgrades required during the last long shutdown in order to prepare the detectors for the final HL-LHC phase. This paper is restricted to present the major silicon tracking detector upgrades. In the pixel domain, these are pixel system upgrades performed by ATLAS for the innermost pixel layer in the ongoing shutdown, and the corresponding CMS pixel upgrade in the next shutdown. For the strip domain, both experiments will be installing completely new strip tracker systems in the major detector upgrade for the HL-LHC to enable the experiments to cope with HL-LHC running conditions. At the same time, new pixel detector systems will be installed again, which means that in both ATLAS and CMS completely new silicon systems will be in place for the ultimate high-luminosity regime of the LHC starting around 2024.

\section{Timeline}

The current LHC schedule is characterised by three more LHC runs, with a long shutdown (LS) before each run. The shutdowns are referred to as LS1, LS2 and LS3, with the corresponding detector upgrades labelled Phase-0, Phase-I and Phase-II upgrades. The currently ongoing LS1 will be followed by Run 2, which should see the collection of 75 to $100 \mathrm{fb}^{-1}$ until 2017. After the subsequent shutdown (LS2), which is foreseen to last until spring 2019, another $350 \mathrm{fb}^{-1}$ should be collected in Run 3 by the end of 2021. The final Run 4 should then provide another $3000 \mathrm{fb}^{-1}$ by about 2030. For a more detailed layout of the time plan for the machine and detector upgrades, as well as the physics motivation of the LHC upgrades, both of which are beyond the scope of these proceedings, see [4].

\section{Phase-0 ATLAS IBL}

In this initial LS1 period (2013-2014), ATLAS has scheduled a number of detector consolidation works. The single major ATLAS upgrade activity in Phase-0 is the installation of a new innermost pixel layer, the Insertable B-Layer (IBL) [5, 6] inside the present Pixel detector. The IBL is an additional pixel layer. It is to be inserted between the current innermost pixel layer (the B-Layer) and the beam pipe, as shown in Figure 1. In order to make sufficient room for the IBL, the present beam pipe needs to be replaced with a smaller diameter beam pipe constructed from beryllium.

It is expected that the IBL will improve track and vertex reconstruction and the b-tagging performance, which both are vital to the full exploitation of the physics potential of the ATLAS experiment. The IBL will be made of 14 individual staves, mounted directly on the beam pipe with a tilt angle of $14^{\circ}$. Each IBL stave will combine two sensor technologies: twelve double-chip modules based on planar n-in-n silicon sensors will form the center of the stave; the outer ends of the stave will be equipped with eight modules of 3D silicon sensors which form single chip sized 


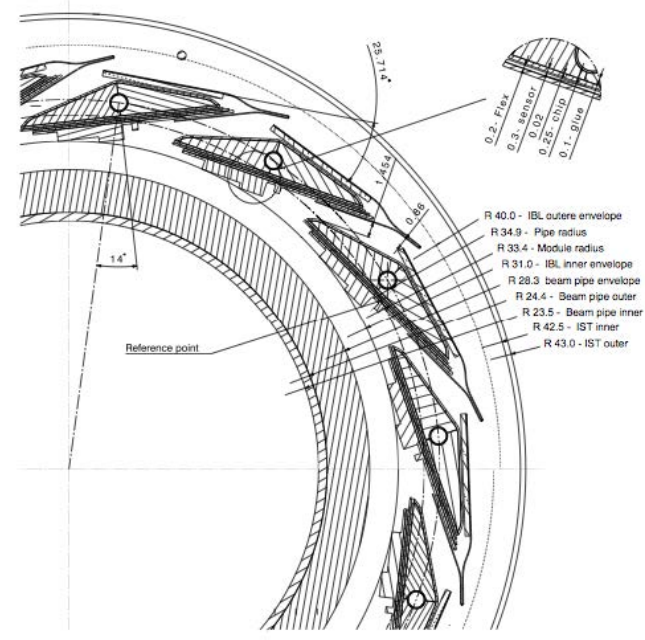

Figure 1: Sketched layout of the ATLAS Insertable B-Layer (IBL) (from [7]).

tiles. In parallel, a new front-end readout chip, the FE-I4 [7], has been developed to function at high data transfer rates of up $160 \mathrm{Mb} / \mathrm{s}$. The FE-I4 allows a decrease of the pixel size from the current $50 \mu \mathrm{m} \times 400 \mu \mathrm{m}$ to $50 \mu \mathrm{m} \times 250 \mu \mathrm{m}$.

\section{Phase-I CMS Pixel}

The CMS experiment will install a completely new pixel detector in the winter 2016/17 shutdown. This pixel system is currently being designed and will constitute a major increase in pixel area compared to the present system. It will also (like the ATLAS IBL) have a smaller radius than the present system, reducing the radius of the innermost layer from $39 \mathrm{~mm}$ to $29.5 \mathrm{~mm}$. Figure 2 shows the overall layout of the Phase-I CMS pixel system, and a schematic cross-section of the innermost layer. The CMS pixel upgrade will enlarge the number of barrel layers from three to four, and the number of forward disks from two to three, resulting in an increase of the number of pixels to about 120 Million pixels. In addition, a new read-out ASIC, using the present pixel size of $100 \mu \mathrm{m} \times 150 \mu \mathrm{m}$ but providing less dead time and lower threshold will be employed [8].

\section{HL-LHC ATLAS Inner Tracker}

In both experiments, the massive increase of the instantaneous luminosity to $5 \times 10^{34} \mathrm{~cm}^{-2} \mathrm{~s}^{-1}$ requires a complete replacement of the inner detector due to two main factors: the high occupancy, and the radiation damage associated with the detectors required to withstand an integrated luminosity of $3000 \mathrm{fb}^{-1}$. For ATLAS, the new Inner Tracker (ITK) will be an all-silicon-detector, where the area covered by the current gas-based Transition Radiation Tracker (TRT) will be populated with silicon strip sensors. Figure 3 shows the layout of this all-silicon tracking system, with pixel detectors at the inner radii surrounded by micro-strip layers at larger radii [9]. In the central region, sensors are arranged in cylinders, with four pixel layers followed by three short-strip layers then two long-strip layers. The forward regions will be covered by six pixel disks and seven strip disks. 

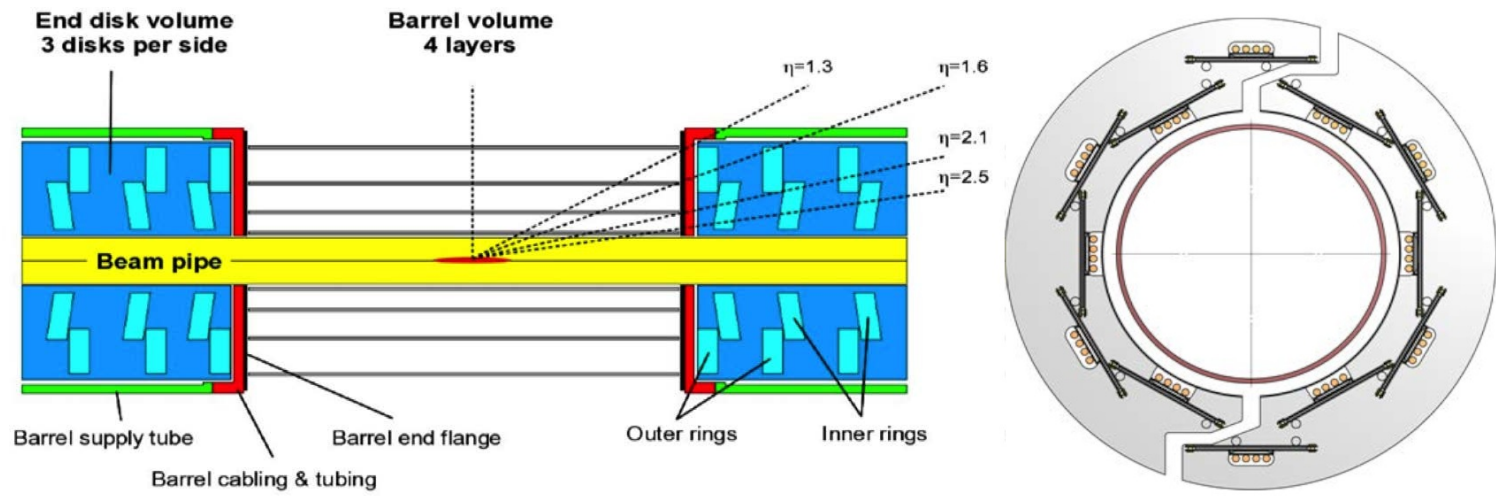

Figure 2: Schematic view of the layout of the CMS Phase-I pixel upgrade (left) and cross-section of the innermost pixel layer (right) (based on [8]).

Strip layers are double-sided with axial strip orientation on one side and sensors rotated by $40 \mathrm{mrad}$ on the other side. In the barrel, two different module types will be used, depending on the radius. The more complicated endcap geometry requires six different module types and up to 18 different variants of front-end hybrids. The most significant modifications with respect to the present inner tracker are replacement of the TRT with $48 \mathrm{~mm}$ long silicon strips; extension of the pixel system out to larger radii; more pixel hits in the forward direction to improve the tracking in this region; and overall smaller pixels and $24 \mathrm{~mm}$ long inner strips to increase the granularity. Also, the layers of silicon are more evenly spaced, especially in the forward region, which is beneficial for track reconstruction.

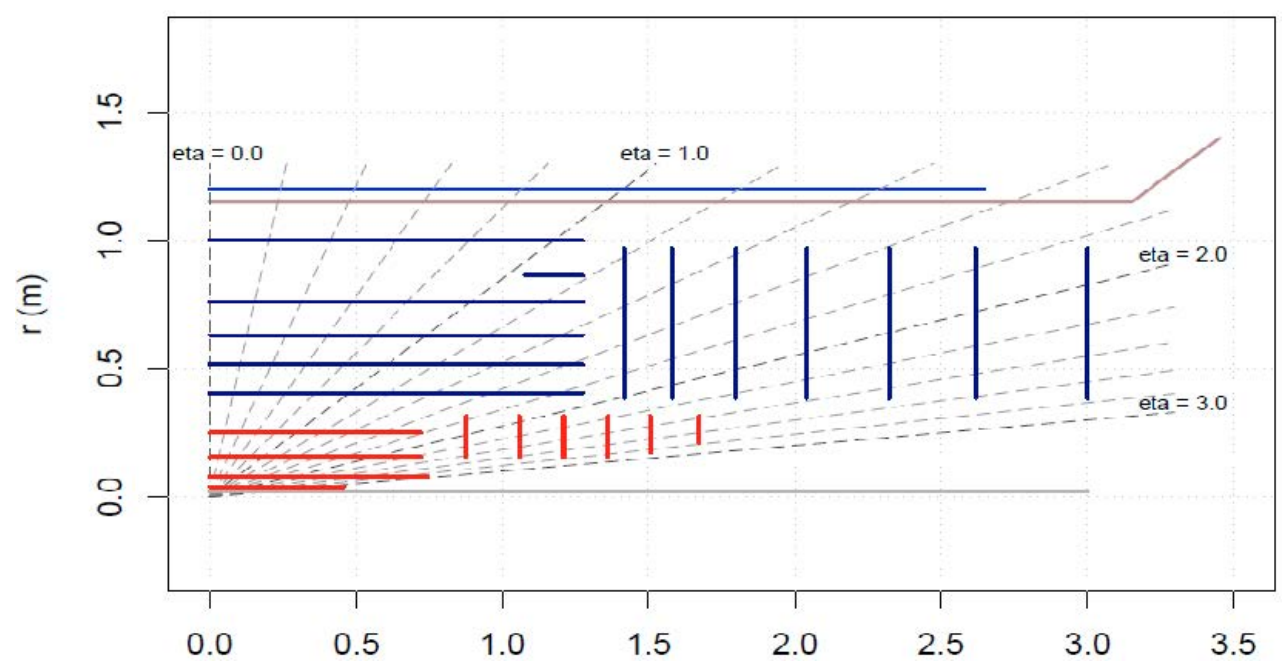

Figure 3: Sketched layout of one quarter of the ATLAS ITK, showing the active areas of the silicon detectors arranged on cylinders and disks (from [9]). The strip sensors appear blue and the pixels sensors appear red.

\section{HL-LHC CMS Inner Tracker}

Like ATLAS, the CMS experiment will install a new all-silicon inner tracker in LS3. At 
present, a number of different design options are studied for the strip detector system for this upgrade. All configurations are foreseen to provide tracks at the hardware level of the trigger. One design, optimised for good tracking performance and low mass, is a conventional design with six barrel layers and seven endcap disks on each side. Another design is characterized by a very long barrel, without a need for endcaps. The modules under study for both designs are double-sided, and use either two strip sensors ( $2 \mathrm{~S}$ modules) in the larger radii, or one strip and one pixel sensor (PS modules) at lower radii [10]. One CMS design under study allows for endcaps to be constructed from the very same two module types as the barrel, which is a considerable simplification compared to the present CMS tracker with 27 module types, and in contrast to the far more complicated ATLAS design. This simplification comes at the cost of a slight increase in radiation length in order to achieve a hermetic coverage with the non-adapted sensor geometry.

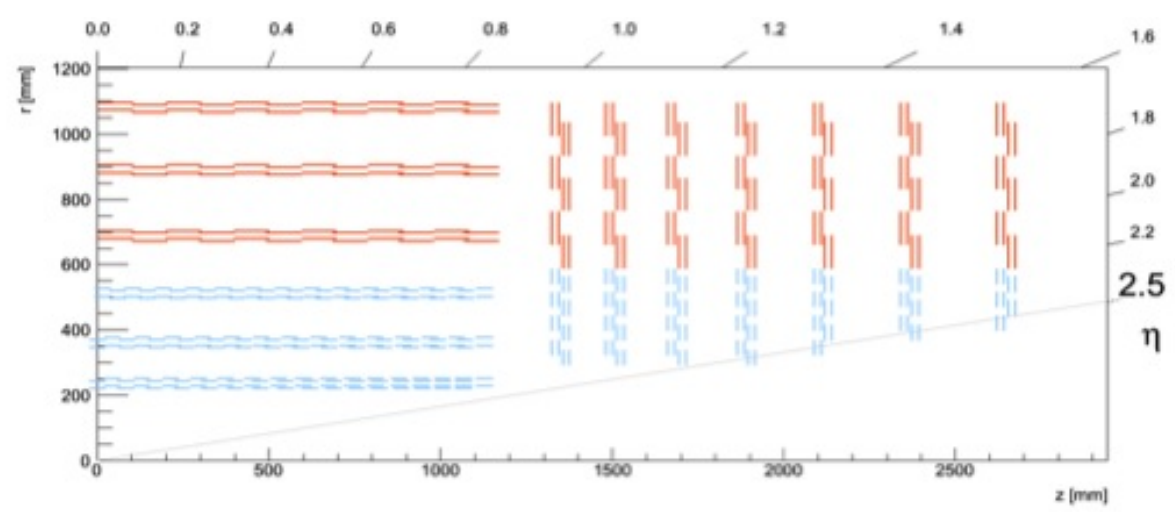

Figure 4: Sketched layout of one quarter of the CMS Phase-II strip tracker (from [10].). The PS modules at lower radii are depicted in blue, the $2 \mathrm{~S}$ modules appear red.

\section{Summary and Conclusions}

Both general purpose LHC experiments ATLAS and CMS are planning a series of detector upgrades synchronized to the LHC upgrade schedule, with a large number of detector installations foreseen during the three long shutdowns. For the inner tracking systems, these upgrades include improvements to the pixel system in Phase-0 (ATLAS IBL) and Phase-I (CMS pixel upgrade), and the major Phase-II upgrades, where in both detectors the entire inner tracker will be replaced with new all-silicon systems.

\section{References}

[1] L. Evans and P. Bryant (editors), 2008 JINST 3 S08001.

[2] ATLAS Collaboration, 2008 JINST 3 S08003.

[3] CMS Collaboration, 2008 JINST 3 S08004.

[4] L. Rossi, O. Brüning et al. High-Luminosity Large Hadron Collider; A description for the European Strategy Preparatory Group. CERN-ATS-2012-236, 2012. 
[5] ATLAS Collaboration, ATLAS Insertable B-Layer Technical Design Report, CERN-LHCC-2010-013.

[6] ATLAS Collaboration, ATLAS Insertable B-Layer Technical Design Report Addendum, CERN-LHCC-2012-009.

[7] The ATLAS IBL Collaboration, Prototype ATLAS IBL modules using the FE-I4A front-end readout chip, JINST 7 (2012) P11010.

[8] CMS Collaboration, CMS TDR for the Pixel Detector Upgrade, CMS UG-TDR-1.

[9] ATLAS Collaboration, Letter of Intent for the Phase-II Upgrade of the ATLAS Experiment, CERN-LHCC-2012-022; LHCC-I-023.

[10] D. Abbaneo, Upgrade of the CMS Tracker with tracking trigger, 2011 JINST 6 C12065. 